\title{
Indoor Microbiome and The Rising Asthma Prevalence
}

\author{
Authors: \\ Xi Fu, ${ }^{1,2} *$ Yu Sun ${ }^{1,3}$ \\ 1. Guangdong Provincial Key Laboratory of Protein Function and Regulation \\ in Agricultural Organisms, College of Life Sciences, South China Agricultural \\ University, Guangzhou, Guangdong, China \\ 2. Department of Occupational and Environmental Health, School of Public Health, \\ Sun Yat-sen University, Guangzhou, Guangdong, China \\ 3. Key Laboratory of Zoonosis of Ministry of Agriculture and Rural Affairs, South \\ China Agricultural University, Guangzhou, Guangdong, China \\ *Correspondence to sunyu@scau.edu.cn \\ Disclosure: $\quad$ The authors have declared no conflicts of interest. \\ Received: $\quad$ 08.11.2019 \\ Accepted: $\quad 03.12 .2019$ \\ Keywords: Asthma, high-throughput sequencing, indoor microbiome. \\ Citation: $\quad$ EMJ Microbiol Infect Dis. 2020;1[1]:51-56.
}

\begin{abstract}
The prevalence of asthma has increased in the past few decades in most developed and developing countries. Large-scale, cross-sectional epidemiological studies have reported several factors associated with asthma prevalence and severity, including parental asthma, tobacco smoking, preterm delivery, virus infection, and air pollution. However, a puzzling problem is that the time trends in the prevalence of these risk factors cannot explain the rise in asthma. For example, the prevalence of smoking and clinical pneumonia have been decreasing globally in the past few decades. Recent progress in high-throughput sequencing technology has promoted the progress of microbiome research and established associations between human and indoor microbiomes, and many metabolic, cognitive, and immune diseases including asthma and allergies. In this review, the authors systematically summarise the current literature, standard practice, and analysis pipeline in the field of indoor microbiome and asthma. The strength and limitation of different analytical approaches are discussed, including the utilisation of relative and absolute abundance in the associated studies. The authors discuss new frameworks of integrated microbiome research from different ecological niches, functional profiling from multiomics data, and how these new insights can facilitate understanding of asthma mechanisms and even the development of new personalised treatment strategies for the rising asthma epidemic.
\end{abstract}

\section{INTRODUCTION}

In the past 50 years the prevalence of asthma, including doctor-diagnosed asthma and asthma symptoms, has been rising in most developing and developed countries and is now one of the most common chronic disorders, affecting $>300$ million patients worldwide., ${ }^{1,2}$ The prevalence of

clinician-diagnosed asthma is generally $>10 \%$ in several developed countries, including the USA, Canada, the UK, and Australia. ${ }^{2}$ In Aberdeen, UK, the proportion of children with asthma increased from 28\% in 1964 and 49\% in 1989 to $64 \%$ in $1999 .{ }^{3}$ The rise can be partly explained by the increased awareness and reporting rate of the disease among the public population, but 
many studies also confirm a surge in asthma symptoms in adults and children. ${ }^{4}$ The rising asthma prevalence has led to huge economic and social costs. In the USA, the annual asthma economic cost has risen from $\$ 12$ billion in 1994 to $\$ 56$ billion in 2011, which includes not only direct costs such as hospitalisation and medication, but also indirect costs such as the inability to work or attend school. ${ }^{5}$ There is a worldwide concern of the prevalence increasing further in the future, and there is a need to identify the risks and protective factors for the global asthma epidemic and propose potential prevention and even personalised treatment strategies for asthma.

\section{Many factors have been reported to be} associated with asthma prevalence and severity. Parental asthma, tobacco smoking, and preterm delivery are suggested to be strong risk factors for paediatric asthma, whereas obesity, birth by caesarean section, respiratory syncytial virus infection, exposure to mould or fungi, and air pollution are also mildly-tomoderately associated with the symptoms. ${ }^{6}$ The prevalence and mechanism is still largely unclear for adult-onset asthma, but risk factors identified so far are generally similar to those of paediatric asthma. For example, a recent largescale, cross-sectional study evaluating asthma prevalence and related risk factors from a large representative general adult population from China reported that cigarette smoking, allergic rhinitis, paediatric pneumonia or bronchitis, and parental respiratory disease were risk factors for adult asthma.7 A perplexing problem is that although the association between these risk factors and the incidence of asthma are wellsupported by many epidemiology studies, the time trends in exposure to these risk factors cannot explain the rise in asthma prevalence. For example, parental or family history of asthma indicates genetic predisposition, and has been identified as one of the most important risk factors for asthma development. ${ }^{6}$ Genome-wide association studies (GWAS) have identified the chromosomal 17q21 region containing ORMDL3 as being strongly associated with both paediatric and adult asthma., ${ }^{8,9}$ However, because the proportion of associated single nucleotide polymorphisms in the human population does not drastically change over a few decades, it is generally accepted that environmental rather than genetic factors are causing the rising prevalence of asthma. Smoking is a clear risk factor for asthma, but the prevalence of smoking decreased globally from 1980 to 2012: from $41.2 \%$ to $31.1 \%$ in men, and $10.6 \%$ to $6.2 \%$ in women. ${ }^{10}$ Similarly, the incidence of clinical pneumonia in young children also decreased by $22.0 \%$ globally over the past two decades. ${ }^{11}$ Most evidence regarding air pollution and allergens from other studies has also failed to explain the elevated asthma prevalence.,12-14 Therefore, there must be other underlying factors related to modern lifestyle contributing to the rising asthma epidemic in the past decades.

\section{INDOOR MICROBIOME AND ASTHMA}

New developments and lower costs in new sequencing technology, as well as their application in culture-free microbial research, have promoted the progress of microbiome research in the past 10 years. Large-scale international collaboration projects, such as the Human Microbiome Project and Earth Microbiome Project, have brought new dimensions to understanding the causes of some prevailing diseases. Numerous other small-scale microbiome studies of specific environmental niches, including those inside or on the surface of humans, animals, and plants, as well as indoor and outdoor environments, have also supplemented our knowledge. ${ }^{15-18}$ New research has identified associations between the human or indoor microbiome and many metabolic, immune, and cognitive diseases, which, similar to asthma, have drastically increased since World War II, including obesity, diabetes, allergies, inflammatory bowel disease, and autism. ${ }^{19}$ These findings shape new concepts for further research. Traditional concepts of public health mainly focus on identifying and understanding the spread of microbes causing infectious diseases and exploring approaches to prevent and treat these diseases. In contrast, the recent microbiome revolution highlights that many microbes inside the human body and indoor environment, previously thought to be of little importance, may be significant in maintaining human health and preventing disease. Instead of conferring risk from the microbes themselves, the loss of microbial diversity and protective microbes in gut and indoor environments, as a 
result of changes in lifestyle, diet, and indoor environment linked to urbanisation, may be contributing to the increased prevalence of allergic, immune, and inflammatory diseases. ${ }^{19}$

The association between microbial composition or diversity in indoor environment and asthma symptoms has been explored in several studies. Population studies in different countries suggest that children who grow up on farms are protected against asthma and atopy. ${ }^{20-22}$ The protection may be due to the wider range of bacterial or endotoxin exposure in the farm environment. ${ }^{20,23,24}$ These findings are conceptually consistent with the hygiene hypothesis proposed by Strachan in 1989.25 The protective effect is suggested to be associated with immunity training and maturation via T-helper cells. ${ }^{20,23,24}$ Similarly, a higher fungal richness, represented by the number of fungal operational taxonomical units (OTU), was also protective against asthma development in a Latino birth cohort study in California, USA. ${ }^{26}$ However, there are also studies suggesting that higher general microbial diversity does not protect against asthma development. Instead, the presence of specific microbes or the farmlike microbial composition have been suggested to be the key factors in reducing indoorrelated asthma development. ${ }^{27,28}$ Two cohort studies in Finland and Germany indicated the farm-like microbial composition, including a higher proportion of Sphingobacteriia, Alphaproteobacteria, and Cyanobacteria, is protective against asthma symptoms. ${ }^{28} \mathrm{~A}$ birth cohort study from the USA found that a relative abundance of 373 bacterial taxa, but not the overall bacterial richness, was associated with childhood asthma. ${ }^{27}$ Furthermore, there are several studies suggesting that high general microbial diversity could be a risk factor for impaired respiratory health. A birth cohort study in Connecticut, USA, and Massachusetts, USA, indicated high bacterial diversity was positively associated with asthma severity. ${ }^{29}$ One study of moisture-damaged buildings also reported that fungal richness, especially for fungal classes Dothideomycetes and Agaricomycetes, was higher in the water-damaged buildings compared to undamaged buildings, suggesting that the high fungal richness could be a potential risk factor for occupants. ${ }^{30}$ It remains an open question whether high microbial richness is a protective factor for development of asthma, or specific taxa or organism compositions may play an important role in asthma and respiratory health.

\section{STANDARD PRACTICE AND TECHNICAL LIMITATION IN INDOOR MICROBIOME STUDY}

Although techniques such as single-strand conformation polymorphism or denaturing gradient gel electrophoresis ${ }^{20,31}$ are still used in microbiome studies of the indoor environment, the majority of current studies use next-generation sequencing techniques to characterise microbiome composition. These new techniques characterise composition by sequencing amplicon genes, including $16 \mathrm{~S}$ ribosomal RNA for bacteria, and $18 \mathrm{~S}$ ribosomal RNA gene or internal transcribed spacer region for fungi. ${ }^{18}$ Several studies have begun to utilise shotgun metagenomics or even metatranscriptomics to characterise bacteria, fungi, viruses, and eukaryotes together. ${ }^{32,33}$ The sequenced amplicon reads are assembled and compared to well-characterised and curated reference datasets such as Greengenes, Silva, or Unite for taxonomic classification, ${ }^{34}$ with microbial richness then calculated based on the number of OTU identified in each sample. However, if the biomass is highly variable, or if the samples are enriched by one or more highly-abundant taxa, the standard analysing practice may skew the results describing community richness and composition. ${ }^{35}$ If the sequencing depth is not high enough, the high-abundance taxa may swamp the rare taxa and lead to an underestimation of the overall microbial richness in the study sample. These technical limitations may be contributing to the inconsistent associations between microbial diversity and asthma symptoms previously discussed.

To identify potentially-protective or harmful microbes in the indoor environment, researchers have developed different analytical approaches. A standard approach involves screening associations between the relative or absolute abundance of microbes and asthma symptoms. This approach has been used in the majority of published microbiome studies, and has 
identified protective and harmful microbes for asthma in the indoor environment. ${ }^{27,29,31,36}$ The relative abundance is obtained directly from the high-throughput sequencing analysis, revealing the percentage of reads matched for each OTU in the total amount of reads produced. The absolute concentration takes total microbial biomass into consideration; the value is calculated by multiplying the relative abundance for each OTU with the total bacterial or fungal concentration derived from universal primer quantitative PCR. ${ }^{36}$ It is generally accepted that the absolute abundance approach is superior to the relative abundance, ${ }^{36}$ although the relative abundance approach is still used in the majority of microbial association studies. An increase in any one taxon will lead to a decrease in relative abundance of all other taxa, leading to a covariant effect. Thus, the relative approach may fail to link correct microbes to phenotypes and quantitative features, especially for samples with substantial microbial load variation. ${ }^{37}$ One common problem of the relative approach is an overestimation of associated microbes. A methodology paper showed that the number of associated taxa with PM10 mass identified by the relative abundance approach was seven times higher than that identified by absolute abundance. ${ }^{36}$ Another problem is misidentification. One study of the human gut showed that five out of eight taxa identified by the relative abundance approach were false-positive results. ${ }^{37}$ The inconsistencies between the two approaches are generally more severe for samples with drastic biomass variation, but for samples with similar total microbial biomass the relative abundance approach can be comparable with the absolute abundance approach.

A recently-proposed novel analysis framework suggests applying an anchor-based approach to identify beneficial and harmful microbes. ${ }^{28}$ Because the indoor microbiome in the farm environment confers a protective effect for asthma, it can be used as a reference to train a model to predict the protective effect of indoor microbiome in the non-farm environment. This approach has been verified in the Finnish (LUKAS) and German (GABRIELA) birth cohorts. $^{28}$ However, the farm microbiome composition can vary across geographic locations, ${ }^{28,38,39}$ thus the model needs to use the local farm microbiome to predict the protective effect of the urban microbiome. As an example, a LUKAS farm microbiome was used to predict the protective effect of LUKAS urban samples, and a GABRIELA farm microbiome was used to predict GABRIELA urban samples. Thus, future studies should aim to identify the panmicrobiome for the farm environment or a global standardised reference of protective microbiome composition to facilitate a global utilisation of this approach.

\section{INTEGRATED MICROBIOME RESEARCH}

This review is mainly focussed on the indoor microbiome and asthma. However, the microbiome compositions in other ecological niches, such as the human intestine or respiratory tract, may also play important roles in respiratory health. Previous reviews systematically summarised beneficial and harmful microbes in the respiratory tract from several cohort and case-control studies, and demonstrated that healthy microbiota can act as gatekeepers to resist pathogens and protect against development of asthma and other respiratory diseases. ${ }^{40,41}$ Similarly, the composition of the human gut microbiome can not only influence gastrointestinal disease, but also have long-lasting impacts on autoimmune, allergic, and metabolic diseases. ${ }^{42,43}$ The association between gut microbiome and asthma has been demonstrated by several birth cohort studies ${ }^{44,45}$ and mouse experiments. ${ }^{41}$ Feeding Clostridium strains to pathogenfree mice induces regulatory $T$ cells and reduces systemic $\operatorname{lgE}$ production, ${ }^{46}$ whilst a diet with high fibre content changes the ratio of Firmicutes to Bacteroidetes, increasing Th2 cell effector function, which can protect against allergic inflammation in the lung. ${ }^{47}$ Besides direct immunomodulation, gut microbiota can affect asthma in indirect ways. Obesity is a risk factor for asthma, and previous mouse experiments and large-scale microbial genotype-phenotype association studies have linked the composition of human gut microbiota to obesity. ${ }^{48-50}$ Thus, asthma occurrence could be reduced by maintaining a healthy gut microbiota. Furthermore, preterm birth and caesarean section are also risk factors for asthma occurrence. A previous study 
reported that genital tract microbial infection can explain up to $40-50 \%$ cases of preterm births. A recent case-control study revealed that women delivered preterm exhibited lower levels of Lactobacillus crispatus and higher levels of Sneathia amnii and a group of Prevotella species, and these taxa were correlated with proinflammatory cytokines in vaginal fluid. ${ }^{52}$ Previous meta-analysis showed that children born by caesarean section have a $20 \%$ increase in the subsequent risk of asthma. ${ }^{53}$ Reduced vaginal microbial exposure by caesarean section delivery can decrease diversity of gut microbiota, delay Bacteroidetes colonisation, and reduce Th1 responses in the first 2 years of life, contributing to asthma development for the newborns. ${ }^{54}$ Thus, future microbiome studies can integrate marker gene and metagenomics data from different ecological niches, such as the indoor environment and human respiratory tract, gut, and vagina, to facilitate an overall assessment of microbiome exposure and how microbes enter, stimulate, and modulate human immune system activity during asthma development.

\section{References}

1. Eder $\mathrm{W}$ et al. The asthma epidemic. $\mathrm{N}$ Engl J Med. 2006;355(21):2226-35.

2. Anandan $\mathrm{C}$ et al. Is the prevalence of asthma declining? Systematic review of epidemiological studies. Allergy. 2010;65(2):152-67.

3. Devenny A et al. Respiratory symptoms and atopy in children in Aberdeen: questionnaire studies of a defined school population repeated over 35 years. BMJ. 2004;329:489.

4. de Nijs $\mathrm{S}$ et al. Adult-onset asthma: is it really different? Eur Respir Rev. 2013;22(127):44-52.

5. Nunes $\mathrm{C}$ et al. Asthma costs and social impact. Asthma Res Pract. 2017;3:1.

6. Castro-Rodriguez JA et al. Risk and protective factors for childhood asthma: what is the evidence? $\mathrm{J}$ Allergy Clin Immunol Pract. 2016;4(6):1111-22.

7. Huang $\mathrm{K}$ et al. Prevalence, risk factors, and management of asthma in China: a national cross-sectional study. Lancet. 2019;394(10196):407-18.

8. Moffatt MF et al. A large-scale, consortium-based genomewide association study of asthma. $\mathrm{N}$ Engl J Med. 2010;363(13):1211-21

9. Bouzigon $\mathrm{E}$ et al. Effect of $17 q 21$ variants and smoking exposure in early-onset asthma. N Engl J Med. 2008;359:1985-94.

10. $\mathrm{Ng} \mathrm{M}$ et al. Smoking prevalence and cigarette consumption in 187 countries, 1980-2012. JAMA 2014;311(2):183-92.

11. McAllister DA et al. Global, regional, and national estimates of pneumonia morbidity and mortality in children younger than 5 years between 2000 and 2015: a systematic analysis. Lancet Glob Health. 2019;7(1):47-57.

12. Mutius E et al. Prevalence of asthma and atopy in two areas of West and East Germany. Am J Respir Crit Care Med. 1994;149(2):358-64.
13. Lau S et al. Early exposure to house-dust mite and cat allergens and development of childhood asthma: a cohort study. Lancet. 2000;356(9239):1392-7.

14. Cullinan $P$ et al. Early allergen exposure, skin prick responses, and atopic wheeze at age 5 in English children: a cohort study. Thorax. 2004;59(10):855-61.

15. Turnbaugh PJ et al. The human microbiome project. Nature. 2007;449:804-10.

16. Thompson LR et al. A communal catalogue reveals Earth's multiscale microbial diversity. Nature. 2017;551(7681):457-63.

17. Oh J et al. Biogeography and individuality shape function in the human skin metagenome. Nature. 2014:514:59-64.

18. Adams Rl et al. Ten questions concerning the microbiomes of buildings. Build Environ. 2016;109:224-34.

19. Bello $M$ et al. Preserving microbial diversity. Science. 2018;362(6410):334.

20. Ege MJ et al. Exposure to environmental microorganisms and childhood asthma. N Engl J Med. 2011;364(8):701-9.

21. Ege MJ et al. Prenatal exposure to a farm environment modifies atopic sensitization at birth. J Allergy Clin Immunol. 2008;122(2):407-12.

22. von Mutius E, Vercelli D. Farm living: effects on childhood asthma and allergy. Nat Rev Immunol. 2010;10:861-8.

23. Eder W, Mutius E. Hygiene hypothesis and endotoxin: what is the evidence? Curr Opin Allergy Clin Immunol. 2004;4(2):113-7.

24. van Strien RT et al. Microbial exposure of rural school children, as assessed by levels of $\mathrm{N}$-acetylmuramic acid in mattress dust, and its association with respiratory health. J Allergy Clin Immunol. 2004;113(5):860-7.

25. Strachan DP. Hay fever, hygiene, and household size. BMJ. 1989;299(6710):1259-60.

26. Dannemiller $\mathrm{KC}$ et al. Nextgeneration DNA sequencing reveals that low fungal diversity in house dust is associated with childhood asthma development. Indoor air. 2014;24(3):236-47

27. O'Connor GT et al. Early-life home environment and risk of asthma among inner-city children. J Allergy Clin Immunol. 2018;141(4):1468-75.

28. Kirjavainen PV et al. Farm-like indoor microbiota in non-farm homes protects children from asthma development. Nat Med. 2019;25(7):1089-95.

29. Dannemiller $\mathrm{KC}$ et al. Indoor microbial communities: influence on asthma severity in atopic and nonatopic children. J Allergy Clin Immunol. 2016;138(1):76-83.

30. Pitkäranta $M$ et al. Molecular profiling of fungal communities in moisture damaged buildings before and after remediation-a comparison of culturedependent and culture-independent methods. BMC microbiol. 2011;11:235

31. Pekkanen $\mathrm{J}$ et al. Indoor bacteria and asthma in adults: a multicentre casecontrol study within ECRHS II. Eur Respir J. 2018;51(2):pii:1701241.

32. Hsu T et al. Urban transit system microbial communities differ by surface type and interaction with humans and the environment. Am Soc Microbiol. 2016;1(3):e00018-16.

33. Hegarty B et al. Gene expression of indoor fungal communities under damp building conditions: implications for human health. Indoor air. 2018;28(4):548-58.

34. Knight R et al. Best practices for analysing microbiomes. Nat Rev Microbiol. 2018;16(7): 410-22. 
35. Adams Rl et al. A unique signal distorts the perception of species richness and composition in highthroughput sequencing surveys of microbial communities: a case study of fungi in indoor dust. Microbial Ecology. 2013;66(4):735-41.

36. Dannemiller $\mathrm{KC}$ et al. Combining real-time PCR and next-generation DNA sequencing to provide quantitative comparisons of fungal aerosol populations. Atmos Environ 2014;84:113-21.

37. Vandeputte D et al. Quantitative microbiome profiling links gut community variation to microbial load. Nature. 2017;551:507-11.

38. Amend AS et al. Indoor fungal composition is geographically patterned and more diverse in temperate zones than in the tropics. Proc Natl Acad Sci USA. 2010:107(31):13748-53.

39. Barberán $\mathrm{A}$ et al. Continental-scale distributions of dust-associated bacteria and fungi. PNAS 2015;112(18):5756-61.

40. Man WH et al. The microbiota of the respiratory tract: gatekeeper to respiratory health. Nat Rev Microbiol. 2017;15(5):259-70.
41. Huang YJ, Boushey HA. The microbiome in asthma. J Allergy Clin Immunol. 2015;135(1):25-30.

42. Rautava $\mathrm{S}$ et al. Microbial contact during pregnancy, intestinal colonization and human disease. Nat Rev Gastroenterol Hepatol. 2012;9(10):565-76

43. Olszak $T$ et al. Microbial exposure during early life has persistent effects on natural killer T cell function. Science. 2012;336(6080):489-93.

44. Stokholm J et al. Maturation of the gut microbiome and risk of asthma in childhood. Nat Commun. 2018;9:141.

45. Frati $\mathrm{F}$ et al. The role of the microbiome in asthma: the gut-lung axis. Int J Mol Sci. 2018;20(1):123

46. Atarashi $\mathrm{K}$ et al. Induction of colonic regulatory $T$ cells by indigenous Clostridium species. Science. 2011;331(6015):337-41.

47. Trompette A et al. Gut microbiota metabolism of dietary fiber influences allergic airway disease and hematopoiesis. Nat Med. 2014;20(2):159-66.

48. Turnbaugh PJ et al. A core gut microbiome in obese and lean twins. Nature. 2009;457: 480-4
49. Turnbaugh PJ et al. An obesityassociated gut microbiome with increased capacity for energy harvest. Nature. 2006;444(7122):1027-31.

50. Rothschild $D$ et al. Environment dominates over host genetics in shaping human gut microbiota. Nature. 2018;555:210-5.

51. Lamont RF. Infection in the prediction and antibiotics in the prevention of spontaneous preterm labour and preterm birth. BJOG. 2003:110(s20):71-5.

52. Fettweis JM et al. The vaginal microbiome and preterm birth. Nat Med. 2019;25:1012-21.

53. Thavagnanam $\mathrm{S}$ et al. A meta-analysis of the association between caesarean section and childhood asthma. Clin Exp Allergy. 2008;38(4):629-33.

54. Jakobsson HE et al. Decreased gut microbiota diversity, delayed Bacteroidetes colonisation and reduced Th1 responses in infants delivered by caesarean section. Gut. 2014;63(4):559-66.

55. Huan T et al. Systems biology guided by XCMS Online metabolomics. Nat Methods. 2017;14:461-2. 